\title{
Gas-Dynamical Effects of Star Formation
}

\author{
M. P. SAVEDofF \\ Department of Astronomy, University of Rochester, Rochester, New York
}

$A^{7}$ $\mathrm{T}$ the Second Symposium, various contributors discussed the possibility that star formation might provide the energy to maintain the motion field in the interstellar medium. Although Oort and Spitzer ${ }^{1}$ emphasized the importance of the recoil momentum produced by the gradual ionization of the surrounding gas, the important effect appears to be the gas pressure itself when the ionized region is enclosed by the surrounding gas. Indeed, $\mathrm{Kahn}^{2}$ has shown (for onedimensional flow into a vacuum) that the recoil momentum is inefficient in producing mass motion; only a limited range in photon fluxes result in acceleration of neutral hydrogen.

We are led to consider the motions of the ionized region around a newly formed $\mathrm{O}$ star. The result of the high pressure in the ionized region is the formation of compressed neutral hydrogen and of a shock front moving into the undisturbed gas.

It was recognized by Schlüter and Bierman ${ }^{3}$ and by Kahn and Schatzman ${ }^{4}$ that as the HII region expands, it increases in mass. There is the possibility that the resulting ionization of the compressed gas is so rapid that the ionization front catches up with the shock front.

Savedoff and Greene ${ }^{5}$ studied the relative motion of the ionization front assuming that

(a) the pressure jump across the ionization front is negligible;

(b) the ionized gas expands uniformly and isothermally;

(c) radiative cooling results in the neutral compressed region being essentially isothermal (excess energy goes into the radiation field);

(d) the shock front at $10-20 \mathrm{~km} / \mathrm{sec}$ does not lead to dissociation or ionization;

(e) the decelerated motions are instantaneously represented by the similarity solutions obtained by assuming spherical expansion at a constant rate.

With these assumptions the system is probably stable against "self-destruction," once the motion of the ionization front is governed by the rate of expansion. This condition implies that the radius of the ionization

\footnotetext{
${ }^{1}$ J. H. Oort and L. Spitzer, Astrophys. J. 121, 6 (1955).

2 F. D. Kahn, Bull. Astron. Soc. Neth. 12, 187 (1954)

3 A. Schlüter and L. Biermann, Gas Dynamics of Cosmic Clouds edited by H. C. van de Hulst and J. M. Burgers (North Holland Publishing Company, Amsterdam, the Netherlands, 1955), p. 144.

1 E. Schatzman and F. D. Kahn, reference 3, p. 163.

${ }^{6}$ M. P. Savedoff and J. Greene, Astrophys. J. 122, 477 (1955).
}

front is larger than or of the order of the Strömgren sphere radius in the undisturbed gas.

We see in Table $I$ the properties of the motion as a function of time. Here $t$ is in units of $R_{0} / V_{I}$ and is very nearly time since star formation. $R_{0}, R_{S}$, and $R$ are, respectively, the radius of the initial Strömgren sphere, the shock front, and the ionization front. $V_{I}$ is the initial velocity of the shock which at time $t$ has slowed to $V_{S} . M_{C}$ is the mass of the compressed region while $M_{T}$ is the total mass within the shock front.

These predictions can be compared with the observations of systematic motions in the neutral hydrogen, reported at this Symposium by C. Wade around $\lambda$ Orionis and T. K. Menon in Barnard's loop. Both sets of observations imply an expansion about a center. The rates of expansion are reasonably predicted by the model. The observations do not lead to improbable values for the predicted parameters as illustrated using Menon's data. Table II is taken principally from my previously published note. ${ }^{6}$

TABLE I. Model evolution.

\begin{tabular}{ccccc}
\hline \hline$t$ & $R_{S} / R_{0}$ & $R / R_{S}$ & $M_{C} / M_{T}$ & $V_{S} / V_{I}$ \\
\hline 0.00 & 1.0 & 1.0000 & 0.000 & 1.000 \\
0.10 & 1.1 & 0.9998 & 0.134 & 0.931 \\
0.21 & 1.2 & 0.9995 & 0.240 & 0.872 \\
0.46 & 1.4 & 0.9991 & 0.397 & 0.777 \\
0.73 & 1.6 & 0.9987 & 0.507 & 0.703 \\
1.03 & 1.8 & 0.9982 & 0.587 & 0.643 \\
1.35 & 2.0 & 0.9977 & 0.648 & 0.593 \\
2.27 & 2.5 & 0.9965 & 0.748 & 0.503 \\
3.34 & 3.0 & 0.9951 & 0.809 & 0.439 \\
8.98 & 5.0 & 0.9884 & 0.912 & 0.299 \\
\hline \hline
\end{tabular}

TABLE II.

\begin{tabular}{|c|c|c|}
\hline Observed parameters & $\lambda$ Ori & $\begin{array}{l}\text { Barnard's } \\
\text { loop }\end{array}$ \\
\hline Expansion velocity $(\mathrm{km} / \mathrm{sec})$ & 8.5 & 10.0 \\
\hline Radius (pc) & 20 & 60.0 \\
\hline Mass (solar units) & $7.5 \times 10^{4}$ & $10^{5}$ \\
\hline Model parameter & & \\
\hline \multicolumn{3}{|l|}{ Original expansion velocity } \\
\hline (km/sec) (assumed) & 14 & 14 \\
\hline Original Strömgren radius (pc) & 10 & 38 \\
\hline Properties of original star & . & $\begin{array}{c}07.5 \\
M_{v}=-5 \\
M_{\mathrm{Bol}}=9.0\end{array}$ \\
\hline Thermonuclear lifetime & & $1.6 \times 10^{6}$ \\
\hline Age of shell (years) & $1.3 \times 10^{6}$ & $1.9 \times 10^{6}$ \\
\hline Kinetic energy of shell (ergs) & $3 \times 10^{49}$ & $10^{50}$ \\
\hline
\end{tabular}

' M. P. Savedoff, Astrophys. J. 124, 533 (1956). 
Several discrepancies should however be noted:

(a) the observed velocities in Barnard's loop show a small tangential component inexplicable for a spherical model except by rotation (see $\mathrm{T}$. $\mathrm{K}$. Menon, these Proceedings);

(b) the singular high velocity $\mathrm{O}-\mathrm{B}$ stars $\mu$ Columbae, $\mathrm{AE}$ Aurigae, and 43 Arietis seem to originate in this Orion region;

(c) the extended HI compressed region about $\lambda$ Orionis cannot be explained if cooling is efficient. Presumably an adiabatic model would be more appropriate.
In summary we have an observed motion; we have a suggestive mechanism established; we must estimate the energy input into the interstellar medium. If we assume one object of these dimensions per cylindrical volume $500 \mathrm{pc}$ radius and $200 \mathrm{pc}$ high per million years, we obtain an energy input of $6.9 \times 10^{-28} \mathrm{erg} / \mathrm{cm}^{3} \mathrm{sec}$. This figure may be improved with further $21-\mathrm{cm}$ data, but it illustrates that the energy input into the interstellar medium is now an observable quantity, or at least nearly so. Figures thus derived should have precedence over estimates based upon radiation available and efficiency of utilization.

\title{
Gas Dynamics of Galaxy Collisions
}

\author{
M. P. SAVEdofF \\ Department of Physics and Astronomy, University of Rochester, Rochester, New York
}

$W^{2}$ HEN two galaxies collide at extremely high velocities, Baade and Spitzer ${ }^{1}$ note that negligible stellar effects are anticipated but that the interstellar medium is violently affected. Recently the radio source Cygnus A has been identified with what appears to be two colliding galaxies. Baade and Minkowski ${ }^{2}$ describe the optical nebula as two ... "late-type systems, judging by low density gradients of the two disks. Spatially they are oriented face to face, they are slightly decentered, and we look upon them at an angle not far from $45^{\circ}$." " . . . the two systems must be in close contact because of the strong signs of tidal distortion which the nuclei show." The lines are noted as diffuse.

More recently $21-\mathrm{cm}$ neutral hydrogen has been observed from this object by Lilley and McClain ${ }^{3}$ with an almost identical velocity with that of the optical system. They estimate the mass now as $2 \times 10^{9} \mathrm{~A}$ suns $/ \mathrm{kpc}^{2}$ where $A$ is the unknown area of the radio source in square kiloparsecs.

TABLE I.

\begin{tabular}{lccc}
\hline \hline \multicolumn{1}{c}{ Case } & $N$ & $I$ & $M$ \\
\hline Shock velocity & $-167 \mathrm{~km} / \mathrm{sec}$ & $-167 \mathrm{~km} / \mathrm{sec}$ & $-243 \mathrm{~km} / \mathrm{sec}$ \\
Compression $\rho_{a} / \rho_{i}$ & 4 & 4 & 3.06 \\
Newtonian sound & $289 \mathrm{~km} / \mathrm{sec}$ & $204 \mathrm{~km} / \mathrm{sec}$ & $178 \mathrm{~km} / \mathrm{sec}$ \\
$\begin{array}{l}\text { speed } \sqrt{ } / \rho \\
\text { Equiv. temperature }\end{array}$ & $10^{7} \mathrm{~K}$ & $\ldots \times 10^{\circ} \mathrm{K}$ & $3.8 \times 10^{60} \mathrm{~K}$ \\
Initial $H^{2} / 8 \pi P$ & $\cdots$ & $\cdots$ & $2.1 \times 10^{4}$ \\
$\begin{array}{l}\text { Final } H^{2} / 8 \pi P \\
\text { Average energy per } \\
\text { particle }\end{array}$ & $\cdots$ & $\cdots$ & 1 \\
\hline \hline
\end{tabular}

1 W. Baade and L. Spitzer, Astrophys. J. 113, 413 (1951).

${ }^{2}$ W. Baade and R. Minkowski, Astrophys. J. 119, 206 (1954).

${ }^{3}$ A. E. Lilley and E. F. McClain, Astrophys. J. 123, 172 (1956).
The model considered assumes we have two identical layers of gas of density $n=1 \mathrm{~cm}^{-3}$, temperature $T=100^{\circ} \mathrm{K}$, each of a width of $200 \mathrm{pc}$, and finally a relative velocity of $10^{3} \mathrm{~km} / \mathrm{sec}$. These layers are presumably stabilized gravitationally against expansion. For a one Bohr radius atomic cross section, these objects are each $6 \times 10^{4}$ Bohr free paths thick, hence can be treated by fluid dynamics.

Kahn's treatment of this problem appears to be valid only for the first instants of mutual contact of these galaxies, if such a time has any meaning for such a diffuse collision. After about $10^{8}$ seconds we may expect to find a compressed hot gas, taken as being at rest, containing the initial contact surface $(C)$ between the gas derived from the two galaxies, two shocks $(S)$ moving out into the oncoming gas, and finally the undisturbed gas coming in at $500 \mathrm{~km} / \mathrm{sec}$ from either side.

Applying the Rankine-Hugoniot conditions across the shocks (or the de Hoffman-Teller ${ }^{4}$ conditions for transverse magnetic fields) we obtain the estimates of Table I for three cases: $(N)$ no ionization; $(I)$ complete ionization; $(M)$ ionization with a final magnetic pressure equivalent to the gas pressure present (includes no fields in vacuum between gas clouds).

We can estimate the rate of ionization of the hydrogen at these energies. Calculations by Bates and Griffing ${ }^{5}$ for the reaction $H(1 s)+H(1 s) \rightarrow H^{+}+e+H$ permit the estimate that the cross section exceeds $0.1 \pi a_{0}^{2}$ in the range $500 \mathrm{ev}$ to $1000 \mathrm{ev}$. Thus collisional ionization

${ }^{4}$ F. de Hoffman and E. Teller, Phys. Rev. 80, 692 (1950).

${ }^{5}$ D. R. Bates and G. W. Griffing, Proc. Phys. Soc. (London) A68, 90 (1955). 